\title{
ORIGINAL
}

\section{CONCEPTUACIÓN DE LA FIBROMIALGIA: ¿CONSENSO O DISCREPANCIA ENTRE PROFESIONALES CLÍNICOS DE ESPAÑA? ${ }^{(*)}$}

\author{
Rubén Uclés-Juárez (1), David Fernández-Carreño (1), Sergio Fernández-Miranda López (2) y Adolfo J. Cangas Díaz (1) \\ (1) Departamento de Psicología. Universidad de Almería. Almería. España. \\ (2) Complejo Hospitalario Torrecárdenas. Almería. España.
}

Los autores declaran que no existe conflicto de intereses.

(*) Financiación: Este estudio ha sido financiado por el Ministerio de Educación, Cultura y Deporte (FPU2013/01660).

\section{RESUMEN}

Fundamentos: El abordaje clínico de la fibromialgia es causa de frustración para algunos profesionales por la controversia en su diagnóstico, etiopatología y manejo clínico. El objetivo del presente estudio fue explorar cuáles son las creencias y conocimientos hacia la fibromialgia por parte de profesionales de distintas especialidades del ámbito público de salud de la provincia de Almería.

Métodos: Se trató de un estudio descriptivo y transversal. Se aplicó un cuestionario elaborado ex profeso a una muestra de 103 clínicos de Atención Primaria, Salud Mental, Medicina Interna y Reumatología. Se exploró la relación de las variables mediante la prueba chi-cuadrado.

Resultados: El 59,7\% de los profesionales de Atención Primaria y el 66,7\% de los de Medicina Interna conceptuaron la fibromialgia como una somatización. Asimismo, el 58,9\% de los profesionales de Atención Primaria indicó que en ningún caso había de considerarse como una enfermedad discapacitante, en comparación con el 16,7\% de los de Reumatología, así como que para el $42,5 \%$ no era fundamental su diagnóstico, en comparación a la totalidad en otras especialidades. Los trastornos del ánimo, como entidades de las que resulta difícil de discriminar la fibromialgia, fueron mencionados por el $53,4 \%$ de los de Atención Primaria. Los problemas de sueño o problemas gastrointestinales se mencionaron en mayor medida como manifestaciones clínicas entre los de Reumatología ( $83,3 \%$ y $50 \%$, respectivamente).

Conclusiones: Un alto porcentaje de profesionales conceptúan la fibromialgia como una entidad psicógena, en especial, profesionales de Medicina Interna y Atención Primaria, destacando en esta última una posición en contra de la consideración de discapacidad y su difícil diferenciación de los trastornos del ánimo. Estos últimos hallazgos podrían explicarse por creencias o actitudes respecto a la amplificación de las conductas del dolor y la obtención de ganancias secundarias. Destacan los conocimientos más profundos de la sintomatología en Reumatología. Se discuten las limitaciones metodológicas y recomendaciones.

Palabras clave: Fibromialgia, Dolor crónico, Creencias, Actitudes, Conocimientos, Percepción, Manejo clínico, Profesionales de la salud, Médicos, Atención primaria.

Correspondencia:

Rubén Uclés Juárez

Departamento de Psicología

Universidad de Almería

Carretera Sacramento, s/n

04120 La Cañada de San Urbano, Almería, España

ruj501@ual.es

\section{ABSTRACT \\ Conceptuation on fibromyalgia: consensus or discrepancy among healthcare providers from Spain?}

Background: The clinical approach of fibromyalgia is a cause of frustration for some professionals due to the controversy on its diagnosis, etiopathology and clinical management. The aim of this study was to explore the beliefs and knowledge on fibromyalgia among health professionals from different specialties of the public health service from Almería province.

Methods: A descriptive and cross-sectional study was carried out. A questionnaire prepared $e x$ profeso was applied to a sample of 103 clinicians from Primary Care Physicians, Mental Health, Internal Medicine and Rheumatology. The chisquare test was performed to explore the relationships among variables.

Results: $59.7 \%$ of Primary Care providers and $66.7 \%$ of Internal Medicine conceptualized fibromyalgia as a somatization. Likewise, $58.9 \%$ of Primary Care indicated that fibromyalgia should be considered in no case as a disabling illness, compared to $16.7 \%$ from Rheumatology, as well as for $42.5 \%$ of them the fibromyalgia diagnosis is not essential, compared to the amount of other specialties. Mood disorders were mentioned by $53.4 \%$ of Primary Care as entities from which fibromyalgia is difficult to discriminate. Sleep or gastrointestinal problems were mentioned by $83.3 \%$ and $50 \%$ of Rheumatology professionals, respectively.

Conclusions: A high percentage of professionals considered fibromyalgia as a psychogenic entity, especially among Internal Medicine and Primary Care, highlighting in the last one a position against the consideration of disability and its difficult differentiation from mood disorders. These latest findings could be explained by beliefs or attitudes on the amplification of pain behaviors and secondary gains. A deeper knowledge on fibromyalgia symptoms from Rheumatology is highlighted. Methodological limitations and recommendations are discussed.

Key words: Fibromyalgia, Chronic pain, Beliefs, Attitudes, Knowledge, Perception, Clinical management, Healthcare providers, Physicians, Primary care.

Cita sugerida: Rubén Uclés-Juárez R, David FernándezCarreño D, Fernández-Miranda López S, Cangas Díaz AJ. Conceptuación de la fibromialgia: ¿consenso o discrepancia entre profesionales clínicos de España? Rev Esp Salud Pública. 2020; 94: 10 de enero e202001006. 


\section{INTRODUCCIÓN}

La fibromialgia (en adelante, FM) es un síndrome idiopático crónico cuyo síntoma principal es dolor generalizado y difuso ${ }^{(1)}$. Otros síntomas incluidos en sus criterios diagnósticos son la fatiga, problemas relacionados con el sueño o descanso, problemas cognitivos, dolor de cabeza, dolor abdominal y depresión ${ }^{(1)}$. En el ámbito epidemiológico, afecta al $2,4 \%$ de la población española mayor de 20 años, y en su mayoría a mujeres ${ }^{(2)}$, lo que supone un elevado gasto socioeconómico en costes directos e indirectos ${ }^{(3)}$. Su manejo clínico es causa de frustración para los profesionales, ya que esta población a menudo reportan una mejoría limitada en dolor y funcionamiento con los tratamientos biomédicos convencionales ${ }^{(4,5,6)}$. Solo los pacientes con fallo renal terminal tienen peor calidad de vida ${ }^{(6)}$.

Para algunos profesionales, su procedimiento diagnóstico resulta controvertido dada la falta de signos "objetivos", cuestionándose que sea una condición médica diagnosticable ${ }^{(7)} \mathrm{y}$ plenamente diferenciable de otras ${ }^{(6,8)}$. Así, existe una percepción generalizada de falta de conocimiento y habilidad para identificarla ${ }^{(7,9)}$. En Perrot et $\mathrm{al}^{\left({ }^{(9)}\right.}$, un estudio mundial que exploraba la percepción de distintos especialistas médicos, se encontró que los reumatólogos mostraban mejores conocimientos en el diagnóstico de FM que profesionales de otras especialidades y de Atención Primaria. Sin embargo, en Hayes et $\mathrm{al}^{(6)}$, médicos de Atención Primaria franceses percibían tener más habilidad en su diagnóstico y mayor confianza en los criterios diagnósticos que en otras especialidades, incluida Reumatología.

Asimismo, se ha discutido la utilidad del diagnóstico, en cuanto al papel que juega el constructo en el control de la conducta del paciente $-y$ del profesional-. Algunos autores sostienen que la propia etiqueta predetermina el "papel de enfermo"(10), lo que se podría traducir en mayor presencia de ideas catastrofistas acerca de la enfermedad -habituales en $\mathrm{FM}^{(11)}$ - y otras conductas de dolor ${ }^{(12,13,14)}$. Así, algunos entienden que ese papel o etiqueta diagnóstica tiene mayor prevalencia en las mujeres, no solo por las diferencias de género atribuidas en términos de manifestación de las emociones, conducta y búsqueda de atención médica, sino porque también goza de mayor aceptación social ${ }^{(15)}$. Con todo, algunos profesionales dudan de la autenticidad de la experiencia del enfermo. En nuestro país, algunos de estos profesionales han reportado que ciertas pacientes con FM sobreactúan para recibir el diagnóstico por las ganancias secundarias que implica reconocer su sufrimiento en forma de enfermedad médica, como bien se mostró en el estudio de BrionesVozmediano et $\mathrm{al}^{(15)}$. En la misma línea, en Santamaría et a ${ }^{(16)}$ fue percibida como la segunda patología con mayor incidencia de simulación, por delante de la depresión, el traumatismo craneoencefálico y la lumbalgia.

Si bien el origen de la FM es desconocido, se han sugerido multitud de factores desencadenantes de distinta índole ${ }^{(17,18,19,20,21)}$. Por un lado, se cree que la FM es un Síndrome de Sensibilización Central, debido a los distintos hallazgos a nivel neurofisiológico ${ }^{(22)}$. No obstante, hasta el momento se desconoce el papel que desempeñan dichos correlatos, en tanto que no sabemos si son causa o consecuencia ${ }^{(23,24)}$.

Otras posiciones entienden la FM como un problema fundamentalmente "psicógeno", y que no es más que una nueva conceptuación desde la Reumatología de otras entidades ya definidas (por ejemplo, trastornos somatoformes) ${ }^{(24,25)}$. En un estudio con reumatólogos canadienses, más de la mitad creía que la FM es de naturaleza psicógena en lugar de física ${ }^{(26)}$. Sin embargo, en otros estudios con estos especialistas, se han hallado menores porcentajes ${ }^{(27)}$. En nuestro país, Pastor et al ${ }^{(28)}$ encontraron que en torno al $60 \%$ de los médicos de Atención Primaria atribuyen 
la FM a factores psicológicos, como la personalidad, el estrés o eventos adversos.

Respecto a los tratamientos que presentan mayor evidencia, según estudios de meta-análisis acerca de las guías de manejo clínico de la FM, son el ejercicio físico, la terapia cognitivo conductual, la amitriptilina de forma puntual y la terapia multi-componente ${ }^{(29,30)}$. Sin embargo, en la práctica clínica no todos los profesionales siguen estas indicaciones por diferentes razones (creencias o desconocimiento, falta de recursos, presiones laborales, falta de entrenamiento, etc.), abordándose la FM principalmente desde un enfoque biomédico o farmacológico. Extendido en el tiempo, este enfoque puede resultar inútil cuando no contraproducente ${ }^{(31)}$, generando otros síntomas que pueden pasar por los de la propia $\mathrm{FM}^{(32,33)}$. Además, no existe unanimidad entre los profesionales sobre qué especialidad médica ha de desempeñar el rol principal en su manejo. En Ghazan-Shahi et al ${ }^{(26)}$ se observó que para el 70\% de reumatólogos canadienses, la Reumatología no debería desempeñar el papel principal en su abordaje, y el $89 \%$ opinó que debería desempeñarlo el médico de familia.

En la literatura existen pocos estudios que exploren la conceptuación de los profesionales clínicos sobre la FM en España ${ }^{(15,16,28)}$. Asimismo, la mayoría de los estudios disponibles exploran las percepciones de los reumatólogos y, en menor proporción, las de otras especialidades. Debido al carácter novedoso y a la importancia que pueden jugar las percepciones en la regulación de la conducta del clínico ${ }^{(34)}$, el objetivo del presente estudio fue explorar cuáles son las creencias y conocimientos respecto a la fibromialgia en los profesionales de la salud de distintas áreas o especialidades clínicas.

\section{SUJETOS Y MÉTODOS}

Diseño y muestra del estudio. Se trató de un estudio descriptivo, ex post facto y transversal ${ }^{(45)}$.
La muestra constó de 103 profesionales médicos de distintas especialidades y de psicólogos del ámbito sanitario público de la provincia de Almería. Los criterios de inclusión fueron ser profesional clínico del Servicio Andaluz de Salud público y pertenecer a las especialidades de Atención Primaria, Psicología Clínica, Medicina Interna y Reumatología. Para su selección se siguió un muestreo no probabilístico por conveniencia. La media de edad de los participantes fue de 44,8 años (DT=12,524), siendo el $39,8 \%$ hombres y el $60,2 \%$ mujeres. El promedio de años de experiencia fue de 18,76 (DT=11,57), y el 93,21\% indicó tener experiencia en el manejo clínico de pacientes con fibromialgia.

La tabla 1 muestra los valores de cada una de las variables sociodemográficas por grupos de Unidades de Gestión Clínica: Atención Primaria (AP), Salud Mental (SM), Medicina Interna (MI) y Reumatología (RE). En cuanto a la distribución de la muestra según especialidad clínica, un 70,9\% fueron médicos de Atención Primaria, un 16,4\% psicólogos clínicos y psiquiatras de salud mental ( $8,7 \%$ y $7,7 \%$, respectivamente), un $5,8 \%$ reumatólogos, y un $6,8 \%$ médicos internistas. El 26,21\% trabajaban en centros de salud de zonas rurales y el $73,78 \%$ en centros de salud u hospitales de ámbito urbano. Asimismo, más del 90\% de cada grupo indicó tener experiencia clínica en fibromialgia y una alta proporción de profesionales de SM señaló no tener experiencia en su diagnóstico $(87,5 \%)$.

Instrumento. Se utilizó un cuestionario elaborado exprofeso (Cuestionario de Creencias sobre la fibromialgia, CCF), compuesto de dos partes. En la primera, se incluyeron cuestiones de carácter sociodemográfico, como fueron la edad, sexo, centro o área de trabajo, localidad, profesión y especialidad clínica, experiencia clínica y experiencia en el manejo y diagnóstico de fibromialgia. En la segunda, se indicaron una serie de preguntas de opinión acerca de la fibromialgia (manifestaciones clínicas, 


\section{Tabla 1}

Variables sociodemográficas por grupos de Unidades de Gestión Clínica.

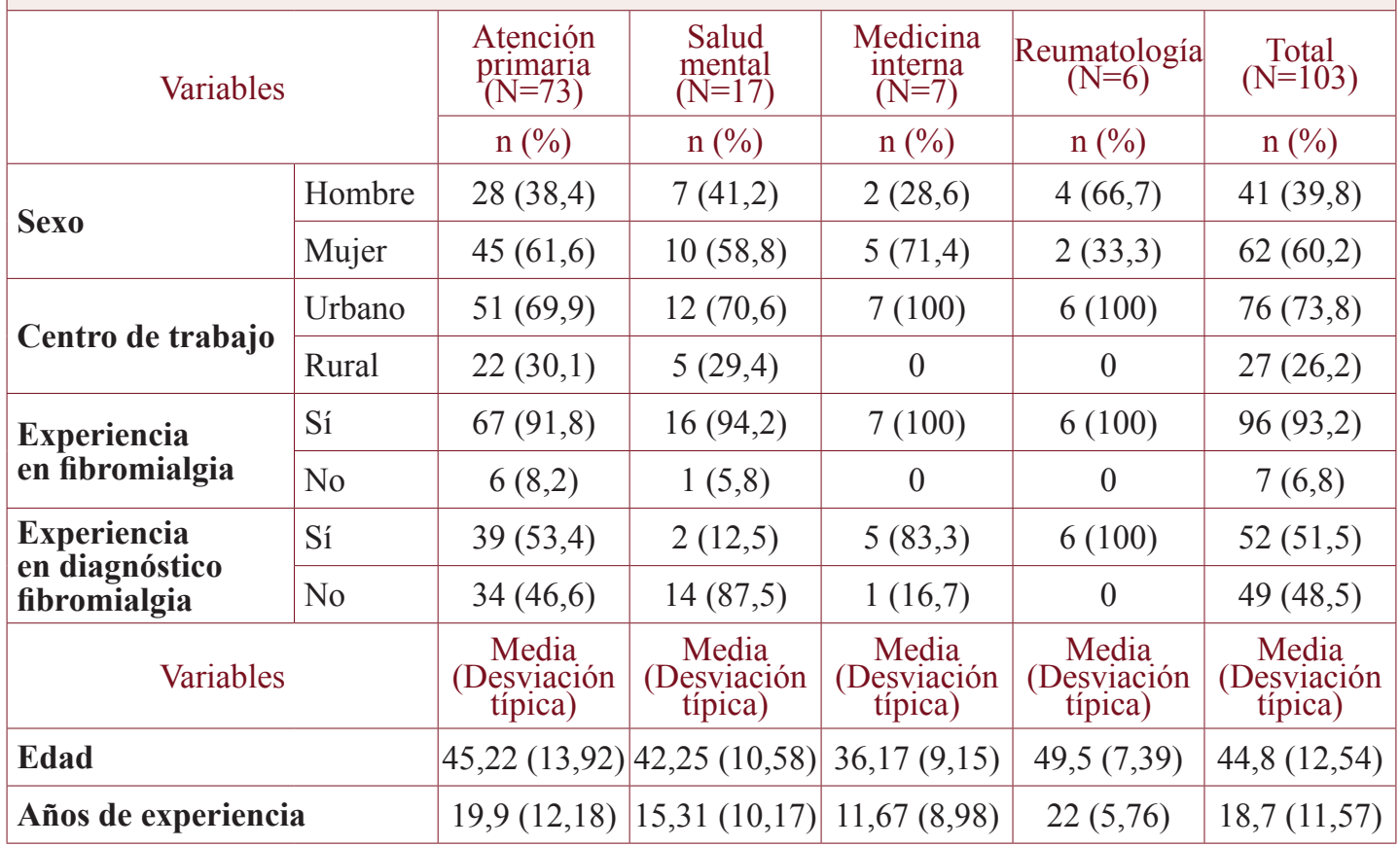

diagnóstico, etiología y abordaje clínico), donde se combinó el formato abierto, el cerrado con respuesta única y el mixto (ver Anexo I).

Procedimiento. Inicialmente, se aplicó una primera versión del cuestionario a 3 médicos y 2 psicólogos para su valoración, ver la utilidad del mismo y depurar las preguntas incluidas. A partir de aquí, se diseñó el cuestionario definitivo. La versión final del cuestionario se incluyó en un sobre con franqueo pagado, junto con el consentimiento informado y una hoja de información del estudio, entregándose a 211 participantes de distintos centros de salud y hospitales públicos de la provincia de Almería, seleccionados de forma discrecional. Antes de acceder a la muestra, se obtuvo el consentimiento del jefe de la Unidad de Gestión Clínica de cada uno de los centros y áreas seleccionadas. Posteriormente, clínicos y residentes colaboradores se encargaron de entregar a los participantes seleccionados el sobre con el cuestionario y la documentación, así como de explicar de forma verbal cualquier duda acerca del estudio. Del total de cuestionarios entregados, se devolvieron al investigador principal y/o colaboradores un total de 133. Finalmente, se incluyeron en el análisis sólo 103 debido a que algunos fueron entregados incompletos o en blanco. La tasa de respuesta del cuestionario debidamente cumplimentado para cada grupo fue la siguiente: 43,97\% de Atención Primaria, 68\% de salud mental, 58\% de médicos internistas y $75 \%$ de reumatólogos.

Categorización de las respuestas. Las respuestas a las cuestiones abiertas o en formato mixto fueron transformadas en variables nominales por parte del equipo de investigación. De esta forma, se crearon categorías para cada cuestión por parte de dos investigadores, tratando de recoger todo 
el espectro de respuestas dadas. Una vez creadas las categorías, dos investigadores asignaron de forma independiente las categorías establecidas a cada una de las respuestas de los participantes. El índice Kappa global interjueces obtenido fue de 0,87 .

Consideraciones éticas. El estudio fue aprobado por el Comité de Bioética de Investigación de la Junta de Andalucía (código de protocolo 0800-N-16).

Análisis estadístico. Se utilizó la prueba chicuadrado para explorar las relaciones entre variables categóricas. Para este análisis y los descriptivos, se utilizó el paquete estadístico SPSS (IBM SPSS Statistics para MacOS, versión
21.0). Asimismo, para la categorización, codificación y cálculo de fiabilidad interjueces de las respuestas abiertas o mixtas se utilizó el programa Microsoft Excel 2018 (versión 16.17).

\section{RESULTADOS}

En cuanto a los resultados de este estudio, en la tabla 2 se pueden ver los resultados de las creencias de cada uno de los grupos (Atención Primaria, salud mental, medicina interna $\mathrm{y}$ Reumatología). Como se observa, existieron diferencias estadísticamente significativas en cuanto a la explicación etiológica de la enfermedad $\left(\chi^{2}=12,793 ; p<0,05\right)$. Destacó el 59,7\% de profesionales de AP y el 66,7\% de MI que indicaron una conceptualización basada en la

Tabla 2

Comparativa de algunas creencias en fibromialgia entre grupos de Unidades de Gestión Clínica.

\begin{tabular}{|c|c|c|c|c|c|c|c|}
\hline \multicolumn{2}{|c|}{ Variables } & $\begin{array}{c}\text { Atención } \\
\text { primaria } \\
n(\%)\end{array}$ & $\begin{array}{l}\text { Salud } \\
\text { mental } \\
\mathrm{n}(\%)\end{array}$ & $\begin{array}{l}\text { Medicina } \\
\text { interna } \\
\mathrm{n}(\%)\end{array}$ & $\begin{array}{c}\text { Reumatología } \\
n(\%)\end{array}$ & $\begin{array}{l}\text { Total } \\
\text { n }(\%)\end{array}$ & $\chi^{2}$ \\
\hline \multirow{3}{*}{$\begin{array}{l}\text { Explicación } \\
\text { etiológica }\end{array}$} & Somatización & $37(59,7)$ & $5(45,5)$ & $4(66,7)$ & 0 & $46(54,10)$ & \multirow{3}{*}{$12,793^{(*)}$} \\
\hline & Orgánica & $20(32,3)$ & $4(36,4)$ & $1(16,7)$ & $3(50)$ & $28(32,95)$ & \\
\hline & Psicosomática & $5(8,1)$ & $2(18,2)$ & $1(16,7)$ & $3(50)$ & $11(12,95)$ & \\
\hline \multirow{3}{*}{$\begin{array}{l}\text { Consideración } \\
\text { de discapacidad }\end{array}$} & Sí, siempre & 0 & 0 & 0 & 0 & 0 & \multirow{3}{*}{$7,144^{(*)}$} \\
\hline & $\begin{array}{l}\text { Sí, dependiendo } \\
\text { de la gravedad }\end{array}$ & $30(41,1)$ & $11(68,8)$ & $5(83,3)$ & $4(66,7)$ & $50(49,50)$ & \\
\hline & $\begin{array}{l}\text { No, en ningún } \\
\text { caso }\end{array}$ & $43(58,9)$ & $5(31,2)$ & $1(16,7)$ & $2(33,3)$ & $51(50,5)$ & \\
\hline \multirow{2}{*}{$\begin{array}{l}\text { ¿Se diferencia } \\
\text { bien de otras } \\
\text { entidades? }\end{array}$} & Sí & $12(16,4)$ & $4(26,7)$ & $1(16,7)$ & $4(66,7)$ & $21(21)$ & \multirow{2}{*}{$8,816^{(*)}$} \\
\hline & No & $61(83,6)$ & $11(73,3)$ & $5(83,3)$ & $2(33,3)$ & $79(79)$ & \\
\hline \multirow{2}{*}{$\begin{array}{l}\text { ¿Cree fundamental } \\
\text { su diagnóstico? }\end{array}$} & Sí & $42(57,5)$ & $9(64,3)$ & $6(100)$ & $6(100)$ & $63(63,64)$ & \multirow{2}{*}{$8,034^{(*)}$} \\
\hline & No & $31(42,5)$ & $5(35,7)$ & 0 & 0 & $36(36,36)$ & \\
\hline \multirow{5}{*}{$\begin{array}{l}\text { Responsable } \\
\text { principal }\end{array}$} & Atención Primaria & $34(46,6)$ & $2(15,4)$ & $4(66,7)$ & 0 & $40(40,81)$ & \multirow{5}{*}{$41,404^{(* * *)}$} \\
\hline & Reumatología & $6(8,2)$ & $5(38,5)$ & 0 & $4(66,7)$ & $15(15,30)$ & \\
\hline & Salud Mental & $15(20,5)$ & $2(15,4)$ & $2(33,3)$ & 0 & $19(19,38)$ & \\
\hline & Medicina Interna & 0 & $2(15,4)$ & 0 & 0 & $2(2,04)$ & \\
\hline & Multidisciplinar & $18(24,7)$ & $2(15,4)$ & 0 & $2(33,3)$ & $22(22,44)$ & \\
\hline
\end{tabular}

(*) $\mathrm{p}<0,05 ;(* * *) \mathrm{p}<0,001$. Valores expresados como frecuencias de. Valores de $\mathrm{p}$ calculados con prueba Chi-cuadrado. 
"somatización" de la FM, frente a la ausencia de profesionales de RE que la conceptuaron así.

Asimismo, contrastó el 16,7\% en MI frente al $50 \%$ en RE tanto para la conceptualización "orgánica" como para la "psicosomática". Haciendo un análisis pormenorizado de estos resultados, si sumamos los porcentajes de la explicación "psicosomática" y de "somatización" en tanto que compartían el carácter psicógeno, destacó el 83,4\% de MI, en contraste con el $50 \%$ de RE.

Respecto a la consideración de "discapacidad", cabe señalar el 58,9\% de profesionales de AP que sostuvo que "en ningún caso ha de considerarse" como una enfermedad discapacitante, en comparación con el 16,7\% de RE $\left(\chi^{2}=7,144 ; p<0,05\right)$. Otro dato no desdeñable es que ningún profesional la consideró discapacitante per se.

También se encontraron diferencias respecto a "si se considera una enfermedad bien diferenciada de otras" $\left(\chi^{2}=8,816 ; p<0,05\right)$. Entre un 73,3 y $83,3 \%$ de profesionales de los grupos de AP, SM y MI indicó no diferenciarla bien de otras enfermedades, en comparación con el $33,3 \%$ de RE.

En cuanto a "si se considera fundamental su diagnóstico", también se encontraron diferencias significativas $\left(\chi^{2}=8,034 ; \mathrm{p}<0,05\right)$. La totalidad de los profesionales de los grupos de MI y RE lo consideraron fundamental, frente al $57,5 \%$ de AP.

Por lo que respecta a "qué especialidades han de llevar el peso principal en el abordaje" de los casos también se encontraron diferencias estadísticamente significativas $\left(\chi^{2}=41,404\right.$; $\mathrm{p}<0,001)$. El 46,6\% de AP, que es la mayoría a nivel intragrupo, se consideró a sí mismo como responsable principal en el abordaje de los casos, considerando en menor medida a
Reumatología como responsable. En contraposición, en RE el 66,7\% se consideró a sí mismo como responsable principal, no otorgando en ningún caso a Atención Primaria dicho papel. Asimismo, el 66,7\% de MI consideraron a Atención Primaria con dicho papel, y salud mental fue considerada en mayor proporción por MI y AP (33,3 y 20,5\%, respectivamente).

Cruzando algunos de estos resultados, no se hallaron diferencias significativas entre grupos en cuanto a la explicación etiológica en función de si creían fundamental $\left(\chi^{2}=9,160 ; p=0,165\right)$ o no $\left(\chi^{2}=0,165 ; p=0,921\right)$ su diagnóstico. Lo mismo sucedió controlando si consideraban que se diferenciaba bien $\left(\chi^{2}=10,417 ; p=0,108\right)$ o no de otras enfermedades $\left(\chi^{2}=7,809 ; p=0,252\right)$.

En cuanto a las razones por las que se consideraba fundamental o no diagnosticar la fibromialgia en pacientes con la sintomatología, se muestran en la tabla 3. Para aquellos que indicaron que sí resultaba fundamental, las razones más frecuentes fueron "para que conozcan su enfermedad" y "para que pueda recibir un tratamiento adecuado" (34,2 y 32,8\%, respectivamente). Para aquellos que no lo consideraban fundamental, las razones más frecuentes fueron "por problemas de constructo" (48,7\%) y "porque genera estigma" (21,9\%), siendo la primera de ellas mencionada en mayor medida por AP y SM (20\% y 25\%, respectivamente), frente a la no mención en los otros dos grupos. Sin embargo, estas diferencias no fueron estadísticamente significativas $\left(\chi^{2}=4,433 ; p=0,193\right)$, como así lo fue con el resto de las razones mencionadas por los profesionales.

En la tabla 4 se muestran otras cuestiones acerca del diagnóstico y tratamiento de la FM, ordenadas según frecuencia de mención. En lo que respecta a las entidades de las que la fibromialgia resulta difícil diferenciar, la más frecuente fue la de "trastornos del ánimo" (37\%), seguida de "enfermedades reumáticas" 


\section{Tabla 3}

Razones a favor o en contra de diagnosticar la fibromialgia.

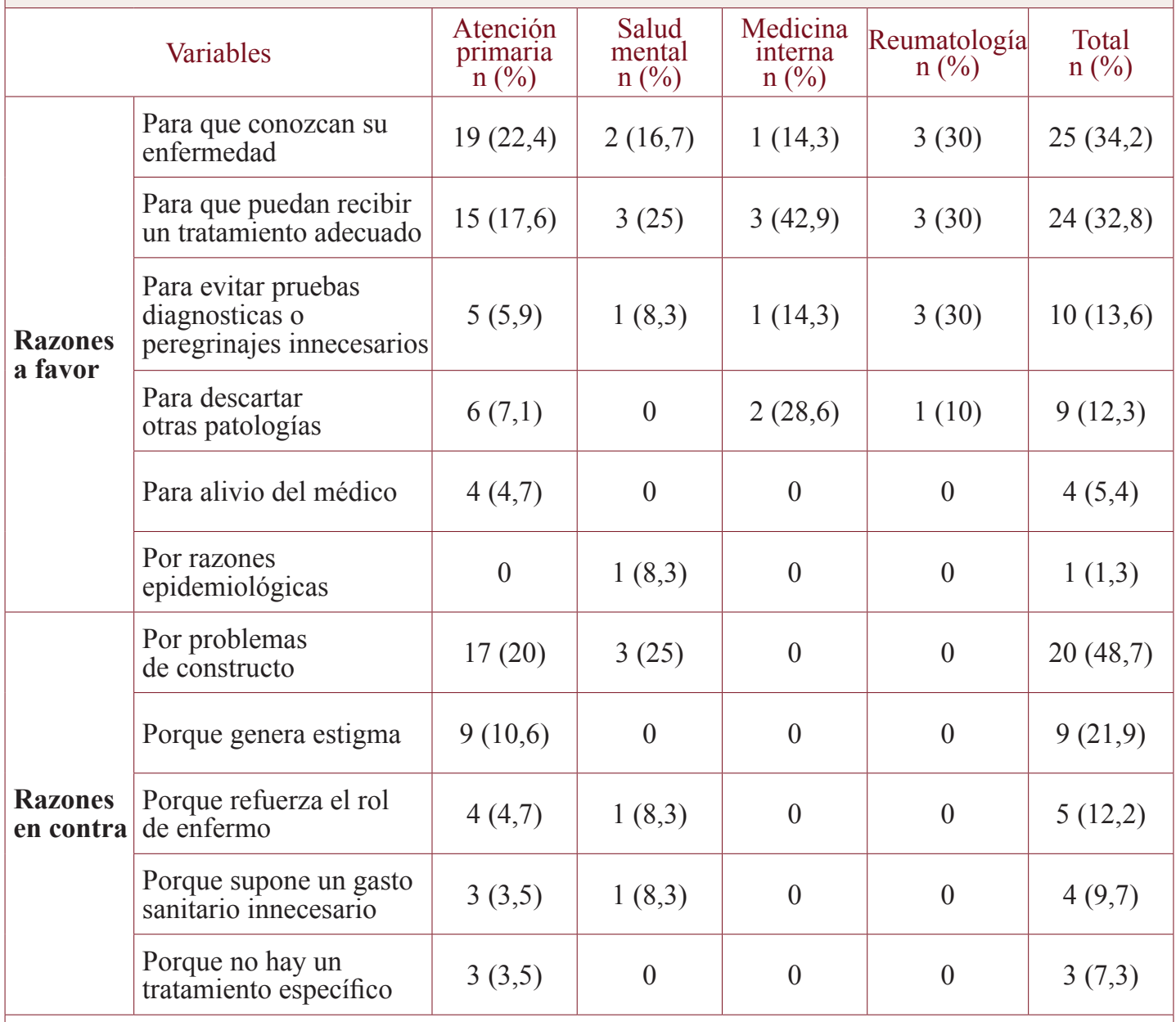

(*) $\mathrm{p}<0,05$. Valores expresados como frecuencias de mención por cada grupo de participantes. Valores de $\mathrm{p}$ calculados con prueba Chi-cuadrado. Se aportaron entre 1 y 2 razones por cada uno de los participantes que aportaron razones a favor o en contra de su diagnóstico.

$(23,5 \%)$. La menos frecuente fue la de "trastornos de síntomas somáticos" (3,4\%). En cuanto a las diferencias entre grupos, se hallaron diferencias significativas en frecuencia de mención de "trastornos del ánimo" $\left(\chi^{2}=12,605\right.$; $\mathrm{p}<0,001)$, siendo más frecuente en $\operatorname{AP}(53,4 \%)$ y RE (33,3\%). Asimismo, aún no encontrándose diferencias significativas, apuntar que en AP se mencionó en menor medida "otros Síndromes de Sensibilidad Central" (5,5\%; $\chi^{2}=5,989$; $\mathrm{p}=0,104)$.

En cuanto a sus manifestaciones clínicas, “Trastornos del ánimo" y "dolor generalizado o difuso" fueron las más frecuentes $(19,7 \% \mathrm{y}$ $18,1 \%$, respectivamente). Por el contrario, las 


\section{Tabla 4}

Otras cuestiones acerca de la fibromialgia.

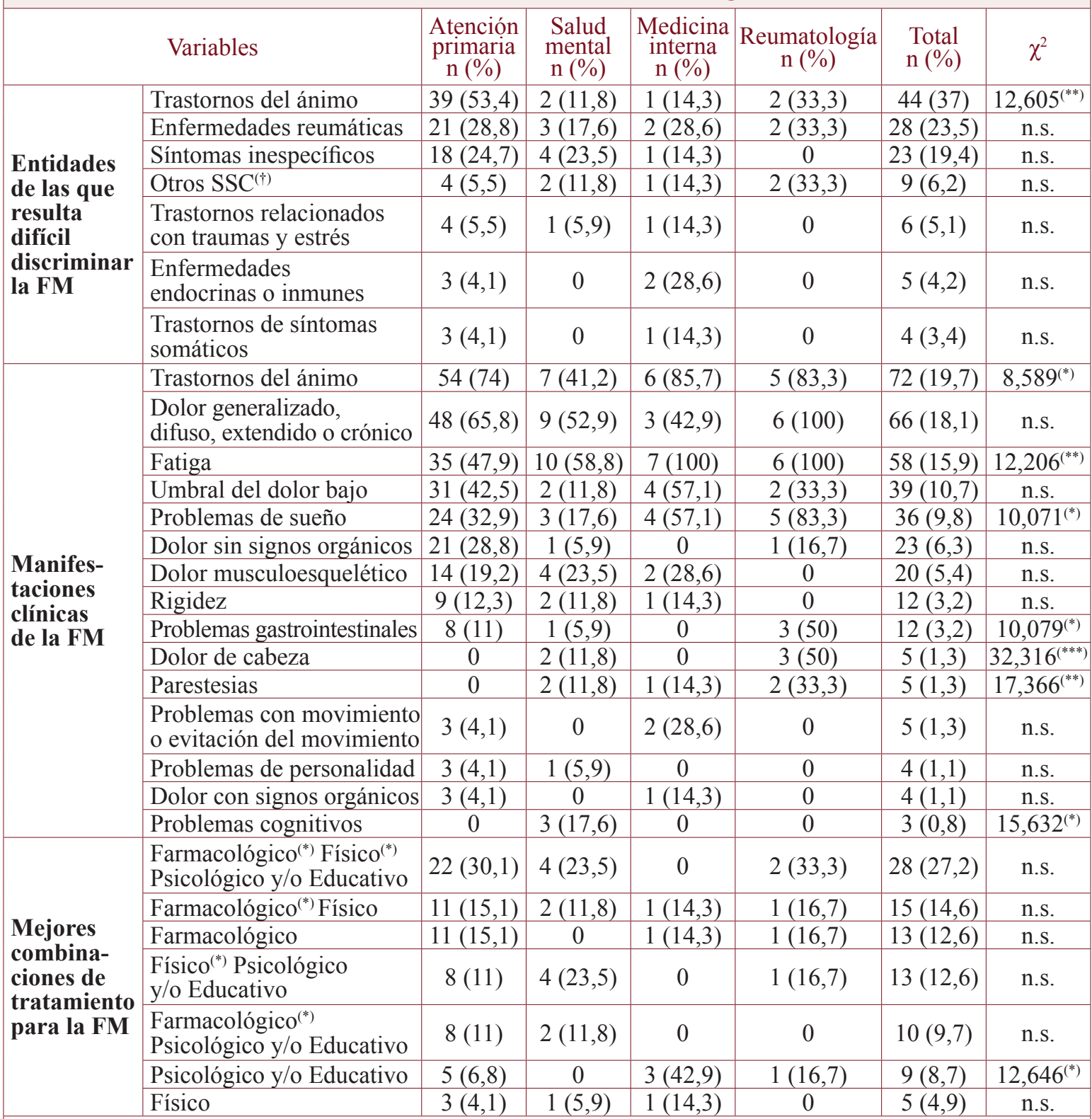

(*) $\mathrm{p}<0,05 ;(* *) \mathrm{p}<0.01 ;(* * *) \mathrm{p}<0.001$. Valores expresados como frecuencias de mención por cada grupo de participantes. Valores de p calculados con prueba Chi-cuadrado. "Entidades de las que resulta difícil discriminar la fibromialgia": se aportaron entre 1 y 3 entidades por cada uno de los participantes para los que resulta difícil discriminarla de otras. "Manifestaciones clínicas de la fibromialgia": se aportaron entre 1 y 8 manifestaciones por cada uno de los participantes que respondieron a esta cuestión. $(\dagger) \mathrm{SSC}=$ Síndromes de Sensibilidad Central. 
menos mencionadas fueron "dolor con signos orgánicos" y "problemas cognitivos" (1,1\% y $0,8 \%$, respectivamente). Asimismo, se mencionaron en mayor medida y de manera significativa por el grupo de RE las manifestaciones de: "trastornos del ánimo" $\left(83,3 \% ; \chi^{2}=8,589\right.$; $\mathrm{p}<0,05)$, "fatiga" $\left(100 \% ; \chi^{2}=12,206 ; \mathrm{p}<0,01\right)$, "problemas de sueño" (83,3\%; $\chi^{2}=10,071$; $\mathrm{p}<0,05)$, "problemas gastrointestinales" $(50 \%$; $\left.\chi^{2}=10,079, \mathrm{p}<0,05\right)$, "dolor de cabeza" (50\%; $\left.\chi^{2}=32,316 ; \mathrm{p}<0,001\right)$, y "parestesias" (33,3\%; $\left.\chi^{2}=17,366 ; p<0,01\right)$. En cuanto a problemas cognitivos, fueron referidos en mayor medida por SM $\left(17,6 \% ; \chi^{2}=15,632 ; \mathrm{p}<0,05\right)$.

Finalmente, respecto a aquellas modalidades de tratamiento que se consideraban para el abordaje de la FM, la modalidad de tratamiento mencionada con mayor frecuencia fue la que combinaba un "tratamiento farmacológico" con uno "físico y psicológico y/o educativo" $(27,2 \%)$, y la menos mencionada fue aquella con solo "tratamiento físico". También destacar que una intervención exclusivamente "farmacológica" se reportó con mayor frecuencia que aquella "farmacológica" combinada con "psicológico y/o educativo" (12,6 y 9,7\%, respectivamente). Aquí debemos señalar las diferencias entre grupos halladas en un tratamiento "psicológico y/o educativo", siendo citado en mayor medida por MI $\left(42,9 \% ; \chi^{2}=12,646 ; \mathrm{p}<0,05\right)$.

\section{DISCUSIÓN}

La FM resulta una entidad controvertida entre los profesionales que tratan de definirla y comprenderla, como se constata en el presente estudio. En primer lugar, en cuanto a la explicación etiológica de la FM, en su mayoría la conceptúan como una entidad "psicógena", englobándose en esto tanto lo "somatoforme" como lo "psicosomático". En concreto, destaca el alto porcentaje de profesionales de Atención Primaria y medicina interna que la entienden como una "somatización". Sin embargo, y he aquí la mayor discrepancia, es en Reumatología donde menos se conceptúa como una entidad "psicógena", siendo coherente con lo obtenido por Ghazan-Shahi et al ${ }^{(26)}$.

En cuanto a la consideración de discapacidad, es destacable que el 58,9\% de profesionales de Atención Primaria indicaron que "en ningún caso ha de considerarse la discapacidad" en esta población de pacientes. Asimismo, en cuanto a "si es fundamental su diagnóstico", donde mayor controversia existe es entre los profesionales de Atención Primaria. Entre todos aquellos profesionales para los que no se considera fundamental, las razones más frecuentes son "por problemas de constructo" y "porque genera estigma".

Una posible explicación de estos resultados en Atención Primaria es que estos profesionales, al ser los que tienen mayor contacto de forma continuada e información del estado de salud y repertorio de esta población, consideran que el estatus de discapacidad y el propio diagnóstico de la FM resultan contraproducentes en términos de severidad de síntomas. Esto podría ir en la línea de lo apuntado por otros autores $^{(10,12,13,14)}$, quienes consideran que, al ser etiquetada esta enfermedad, queda cosificada en forma de alteración orgánica crónica que daría "explicación” de los síntomas y justificaría el papel de enfermo. De esta forma, la etiqueta posee funciones estigmatizantes y catastrofistas, lo que podría influir en la amplificación de las conductas de dolor. Otra explicación de los resultados es que, para algunos de estos médicos de Atención Primaria, la FM no se trate de una enfermedad sino de falsos pacientes que tratan de obtener ganancias secundarias, como ya se reportó en Santamaría et al y Briones-Vozmediano et al ${ }^{(16,15)}$. También habría de considerarse la existencia de ciertos estereotipos o sesgos de género sobre la base, en tanto que, como se ve en algunos estudios, ciertos profesionales conceptúan el fenómeno 
de determinada forma dependiendo del sexo del paciente, condicionando así el abordaje médico y la consideración de discapacidad ${ }^{(15)}$. Por ejemplo, y en línea con otros autores, conceptuaciones psicógenas del fenómeno se atribuyen en mayor medida cuando el sufridor es de sexo femenino ${ }^{(15)}$.

En lo referente a la cuestión de si se diferencia bien de otras enfermedades, reseñar que la gran mayoría de profesionales no la diferencian claramente de otras, a excepción de Reumatología, donde solo para el 33,3\% no se diferencia de forma clara. Estos datos coinciden con los obtenidos por Perrot et $\mathrm{al}^{(9)}$, donde los reumatólogos mostraron en mayor medida tener una idea clara y mejores conocimientos sobre el diagnóstico de FM que otras especialidades. Asimismo, en línea con lo apuntado por Wolfe et $\mathrm{al}^{(6)}$, las enfermedades o trastornos que más se reportaron en cuanto a la difícil diferenciación con la FM son los "trastornos del ánimo" y las "enfermedades reumáticas", siendo los primeros mencionados con mayor frecuencia por Atención Primaria, en contraste con la menor mención por salud mental. Este hallazgo es esperable, en tanto que en salud mental están a priori más entrenados en reconocer problemas de índole emocional que en Atención Primaria, por lo que diferenciarían con menos dificultades la FM respecto a problemas de corte emocional.

Por lo que respecta a qué especialidades han de llevar el peso principal en el abordaje de los casos, hay que señalar que tanto los médicos de Atención Primaria como los reumatólogos se consideran a sí mismos con ese papel, considerando a los otros en menor medida. Igualmente, medicina interna también considera a Atención Primaria con protagonismo en dicho papel. Estos datos no coinciden con Ghazan-Shahi et al ${ }^{(26)}$, donde la gran mayoría de reumatólogos consideraba que era Atención Primaria la que debería ocupar ese papel. Esta discrepancia puede que tenga que ver con las creencias respecto al origen. Esto sería así puesto que, si se considera en mayor medida por parte de Atención Primaria y medicina interna una somatización y no un problema orgánico, puede que el médico de Atención Primaria, que es el primer profesional que recibe a estas personas, juegue un papel importante en cuanto a la contención de la cronificación y medicalización del problema.

Las manifestaciones clínicas que más se reportan son los "trastornos del ánimo" y "dolor generalizado o difuso". Por el contrario, las menos mencionadas son "dolor con signos orgánicos" y "problemas cognitivos". En su mayoría, los profesionales muestran buenos conocimientos respecto a las manifestaciones clínicas de la FM, según los criterios diagnósticos ${ }^{(1)}$. Sin embargo, los problemas cognitivos, a pesar de estar recogidos en los criterios diagnósticos, apenas se indican como manifestación clínica. Los trastornos del ánimo, fatiga, problemas de sueño, problemas gastrointestinales y parestesias, se mencionan con mayor frecuencia en Reumatología, lo que muestra unos conocimientos más profundos del fenómeno en esta especialidad, siendo coherente con lo obtenido por Perrot et $\mathrm{al}^{(9)}$.

Finalmente, en cuanto a las creencias sobre el tratamiento, la gran mayoría de profesionales cree que la mejor combinación de tratamiento es aquel que implique un "tratamiento farmacológico, físico y psicológico $\mathrm{y} / \mathrm{o}$ educativo", en línea con lo que recomiendan algunas guías de manejo clínico de la $\mathrm{FM}^{(29)}$. Sin embargo, si analizamos con mayor detalle otras combinaciones mencionadas, el tratamiento exclusivamente "farmacológico" se reporta con mayor frecuencia que la combinación de "farmacológico" y "psicológico y/o educativo". Esto contradice a la evidencia, en tanto que el tratamiento farmacológico prescrito de forma aislada resulta poco efectivo para mitigar los 
síntomas $^{(31)}$, además de generar efectos adversos que pueden hacerse pasar por los propios de la $\mathrm{FM}^{(32,33)}$. Es necesario mencionar que un tratamiento exclusivamente "psicológico y educativo" se cita con mayor frecuencia en medicina interna, lo que podría relacionarse con una conceptuación mayoritariamente psicógena del fenómeno entre la muestra de profesionales de la medicina interna.

En definitiva, aún encontrándose diferencias significativas entre grupos, destacar la mayoritaria conceptuación de la fibromialgia como entidad psicógena y su difícil diferenciación de otras entidades entre todos estos profesionales, destacando en particular en Atención Primaria $\mathrm{y}$ medicina interna.

No obstante, los datos aquí aportados han de ser interpretados con cautela, dadas las limitaciones metodológicas del estudio y el carácter descriptivo de los datos. Por un lado, en lo referente al muestreo, este se realizó de forma no probabilística, debido a las dificultades de acceso a la población, hecho que a su vez condicionó el tamaño muestral reducido. Asimismo, la tasa de respuesta fue baja (48,8\%), y en especial, en Atención Primaria, donde se devolvió el 43,97\% de los cuestionarios entregados, contrastando con el $75 \%$ de Reumatología. Esta variabilidad en la disposición a responder a un cuestionario de estas características podría deberse a prejuicios o desinterés de base hacia la entidad.

Por otro lado, aunque las cuestiones empleadas aportan una información valiosa acerca de las creencias y conocimientos, en algunas se echa en falta información más precisa, por lo que sería interesante ampliar estas cuestiones para próximos estudios. Por ejemplo, la cuestión acerca del tratamiento no nos permite explorar de forma específica qué tratamientos se consideran (por ejemplo, tipos de medicamentos) y de qué forma se prescribirían. Además, para próximos estudios se recomienda usar métodos de muestreo probabilísticos de toda la población española con tamaños muestrales más representativos. También sería interesante ahondar en otras creencias a la base que puedan estar condicionando las respuestas al cuestionario (por ejemplo, creencias sobre la salud y la enfermedad, sobre el dolor y su abordaje médico, la consideración de la discapacidad en el rol de enfermo, los sesgos de género, etc.).

\section{AGRADECIMIENTOS}

Los autores agradecen al Complejo Hospitalario Torrecárdenas, al Comité de Bioética de Investigación de la Junta de Andalucía (C.H. Torrecárdenas) y al Distrito Sanitario Almería por el permiso y respaldo durante todo el proceso de estudio. También agradecemos a todos los médicos residentes que colaboraron en la recogida de la muestra (Alejandro, Julia, Marina, Marta, Nerea y Regina).

\section{BIBLIOGRAFÍA}

1. Wolfe F, Clauw DJ, Fitzcharles MA, Goldenberg DL, Häuser W, Katz RL et al. 2016 Revisions to the 2010/2011 fibromyalgia diagnostic criteria. Seminars in Arthritis and Rheumatism. 2016; 46(3): 319-329.

2. Carmona L, Gabriel R, Ballina J, Laffon A y Grupo EPISER. Proyecto EPISER 2000: prevalencia de enfermedades reumáticas en la población española. Revista Española de Reumatología. 2001; 28(1): 18-25.

3. Cabo-Meseguer A, Cerdá-Olmedo G y Trillo-Mata. Fibromialgia: prevalencia, perfiles epidemiológicos y costes económicos. Medicina Clínica. 2017; 149(10): 441-8.

4. Homma M, Ishikawa H y Kiuchi T. Association of physicians' illness perception of fibromyalgia with frustration and resistance to accepting patients: a cross-sectional study. Clinical Rheumatology. 2016; 35(4): 1019-1027.

5. Ruiz-Moral R, Rodríguez-Salvador J, Pérula L, Fernández I, Martínez J, Jesús Fernández M et al. Problemas 
y soluciones en la atención sanitaria de enfermedades crónicas. Un estudio cualitativo con pacientes y médicos. Atención Primaria. 2006; 38(9): 483-9.

6. Wolfe F, Michaud K, Li T y Katz RS. Chronic conditions and health problems in rheumatic diseases: comparisons with rheumatoid arthritis, noninflammatory rheumatic disorders, systemic lupus erythematosus, and fibromyalgia. The Journal of rheumatology. 2010; 37(2): 305-315.

7. Hayes SM, Myhal GC, Thornton JF, Camerlain M, Jamison C, Cytryn KN y Murray S. Fibromyalgia and the therapeutic relationship: Where uncertainty meets attitude. Pain Research and Management. 2010; 15(6): 385-391.

8. Blotman F, Thomas E, Myon E, Andre E, Caubere JP y Taïeb C. Awareness and knowledge of fibromyalgia among French rheumatologists and general practitioners. Clinical and Experimental Rheumatology. 2005; 23(5): 697-700.

9. Perrot S, Choy E, Petersel D, Ginovker A y Kramer E. Survey of physician experiences and perceptions about the diagnosis and treatment of fibromyalgia. BMC health services research. 2012; 12(356): 1-8.

10. Madden S y Sim J. Acquiring a diagnosis of fibromyalgia syndrome: The sociology of diagnosis. Social Theory \& Health. 2016; 14(1): 88-108.

11. Van Ittersum MW, Van Wilgen CP, Hilberdink WKHA, Groothoff JW y Van Der Schans CP. Illness perceptions in patients with fibromyalgia. Patient Education and Counseling. 2009; 74(1): 53-60.

12. Ehrlich GE. Pain is real; fibromyalgia isn't. The Journal of rheumatology. 2003; 30(8): 1666.

13. Hadler NM. Workers' compensation, fibromyalgia, and Kafka. The Journal of rheumatology. 2013; 40: 216-218.

14. Wolfe F. Fibromyalgia Wars. The Journal of Rheumatology. 2009; 36(4): 671-8.

15. Briones-Vozmediano E, Öhman A, Goicolea I y VivesCases C. "The complaining women": health professionals' perceptions on patients with fibromyalgia in Spain. Disability and rehabilitation. 2018; 40(14): 1679-1685.

16. Santamaría P, Capilla Ramírez P y González-Ordi H. Prevalencia de simulación en incapacidad temporal: percepción de los profesionales de la salud. Clínica Y Salud. 2013; 24(3): 139-151.

17. Varinen A, Kosunen E, Mattila K, Koskela T y Sumanen M. The relationship between childhood adversities and fibromyalgia in the general population. Journal of Psychosomatic Research. 2017; 99(4): 137-142.

18. Silberbogen AK, Janke EA y Hebenstreit C. A closer look at pain and hepatitis $C$ : Preliminary data from a veteran population. Journal of Rehabilitation Research \& Development. 2007; 44(2):231-244.

19. Mork PJ y Nilsen TIL. Sleep problems and risk of fibromyalgia: Longitudinal data on an adult female population in Norway. Arthritis and Rheumatism. 2012; 64(1): 281-4.

20. Forseth KO, Husby G, Gran JT y Førre O. Prognostic factors for the development of fibromyalgia in women with self-reported musculoskeletal pain. A prospective study. The Journal of rheumatology. 1999; 26(11): 2458-67.

21. Wynne-Jones G, Macfarlane GJ, Silman AJ y Jones GT. Does physical trauma lead to an increase in the risk of new onset widespread pain? Annals of the rheumatic diseases. 2006; 65(3): 391-3.

22. Jay GW y Barkin RL. Fibromyalgia. Disease-a-Month. 2015; 3(61): 66-111.

23. Wolfe F y Walitt B. Culture, science and the changing nature of fibromyalgia. Nature Reviews Rheumatology. 2013; 9(12): 751 .

24. Walitt B, Ceko ML, Gracely J y Gracely R. Neuroimaging of central sensitivity syndromes: key insights from the scientific literature. Current rheumatology reviews. 2016; 12(1): 55-87. 
25. Barker KK. Listening to Lyrica: contested illnesses and pharmaceutical determinism. Social science \& medicine. 2011; 73(6): 833-842.

26. Ghazan-Shahi S, Towheed T y Hopman W. Should rheumatologists retain ownership of fibromyalgia? A survey of Ontario rheumatologists. Clinical Rheumatology. 2012; 31: 1177-1181.

27. Agarwal A, Oparin Y, Glick L, Fitzcharles MA, Adachi JD, Cooper MD, Busse JW. Attitudes Toward and Management of Fibromyalgia. Journal of Clinical Rheumatology. 2017; 24(5): 243-249.

28. Pastor MA, López-Roig S, Johnston M, Gracia R y Daza P. Clinical self-efficacy and illness beliefs in ambiguous chronic pain conditions: General Practitioners' management of Fibromyalgia. Anales de Psicología. 2012; 28(2): 417-425.

29. Häuser W, Thieme K y Turk DC. Guidelines on the management of fibromyalgia syndrome-a systematic review. European Journal of Pain. 2010; 14(1): 5-10.

30. Busch AJ, Barber KA, Overend TJ, Peloso PMJ y Schachter CL. Exercise for treating fibromyalgia syndrome. Cochrane database of systematic reviews, $2007 ; 4$.
31. Clauw DJ. Fibromyalgia: A clinical review. Journal of the American Medical Association. 2014; 311(15): 15471555. http://doi.org/10.1001/jama.2014.3266.

32. Berthelot JM, Nizard J y Maugars Y. Opioids can paradoxically induce severe pain. Joint Bone Spine. 2018; 85(6): 655-7.

33. Brummett CM, Janda AM, Schueller CM, Tsodikov A, Morris M, Williams DA y Clauw DJ. Survey criteria for fibromyalgia independently predict increased postoperative opioid consumption after lower-extremity joint arthroplastya prospective, observational cohort study. Anesthesiology: The Journal of the American Society of Anesthesiologists. 2013; 119(6): 1434-1443.

34. Darlow B, Fullen BM, Dean S, Hurley DA, Baxter GD y Dowell A. The association between health care professional attitudes and beliefs and the attitudes and beliefs, clinical management, and outcomes of patients with low back pain: a systematic review. European Journal of Pain. 2012; 16(1): 3-17.

35. Montero I y León OG. A guide for naming research studies in Psychology. International Journal of clinical and Health psychology, 2007; 7(3): 847-862. 


\section{Anexo I}

Cuestiones de opinión acerca de la fibromialgia.

Bajo su punto de vista, ¿qué manifestaciones clínicas presentes en un paciente señalarían que se trata de fibromialgia?

\begin{tabular}{|l|l|}
\hline 1 & 5 \\
\hline 2 & 6 \\
\hline 3 & 7 \\
\hline 4 & 8 \\
\hline
\end{tabular}

¿Cree fundamental que se realice el diagnóstico de fibromialgia en pacientes con dichas manifestaciones?

Sí. Indique brevemente las razones:

No. Indique brevemente las razones:

¿Cree que se diferencia bien de otras enfermedades o entidades?

Sí.

No. Indique con cuál/es no se diferencia bien:

En su opinión, ¿existe alguna patología orgánica a la base en fibromialgia?

Sí. Indique qué alteración/es orgánica/s

¿A qué es o son debidas dichas alteraciones?

No. ¿A qué se deben entonces las manifestaciones clínicas de la fibromialgia?

No estoy seguro/a. ¿A qué se aventuraría a decir que se deben las manifestaciones clínicas de la fibromialgia?

¿Piensa que las personas con fibromialgia se han de considerar discapacitadas por las instituciones?

Sí, en cualquier caso

Sí, dependiendo de la gravedad de los síntomas

No, en ningún casoclínicas de la fibromialgia?

¿Cuál es, en su opinión, la que debe ser la responsable principal en el abordaje de los casos?

En su opinión, ¿cuál es el mejor tratamiento (farmacológico y/o no farmacológico) para estos pacientes? 